\title{
Optimisation structurale de problèmes d'amortissement de type shunt résistif
}

\author{
YAnn Meyer $^{1, a}$ et Manuel Collet $^{2}$ \\ 1 Institut Supérieur de Mécanique de Paris (SupMéca Paris), LISMMA/Structure, 3 rue Fernand Hainaut, \\ 93407 Saint-Ouen Cedex, France \\ 2 Institut FEMTO ST dép. MA/UMR 6174, 24 chemin de l'épitaphe, 25000 Besançon, France
}

Reçu le 30 septembre 2008, accepté le 18 février 2009

\begin{abstract}
Résumé - Les besoins accrus d'encapsulation et d'autonomie des dispositifs de contrôle vibratoire ont ouvert le domaine des matériaux piézoélectriques shuntés. De par ses propriétés physiques, l'élément piézoélectrique convertit une partie d'énergie vibratoire en énergie électrique. Le fait de connecter un circuit électrique (un circuit de shunt) aux bornes du patch modifie la dynamique du système complet (structure + patchs piézoélectriques) et offre ainsi des possibilités de le contrôler. C'est ce que nous nommerons contrôle vibratoire passif dans le cas où le circuit de shunt est constitué de composants passifs (Résistances et inductances physiques). Dans la littérature, les dispositifs employant des stratégies de contrôle se focalisent principalement sur l'efficacité obtenue en termes de facteur d'amortissement modal. Les flux d'énergie entre les différents éléments du système complet ne sont pas optimisés. Nous proposons de développer un critère d'optimisation structurale qui prend en compte la dissipation induite non seulement en termes d'amortissement modal mais également en termes d'amplitude d'énergie dissipée. Ce critère intègre des grandeurs facilement calculables via un code de calcul par éléments-finis. Il est à noter que l'une des principales difficultés de modélisation de ce type de structures est liée à la condition électrique qui varie. Il est nécessaire d'employer un modèle complet ou suffisamment représentatif pour calculer les modes de résonance de la structure à tension nulle (condition de Dirichlet électrique ) et à courant nul (condition de Neumann électrique). Nous appliquerons notre critère à une poutre élancée sur laquelle est implantée un patch piézoélectrique pour différentes conditions aux limites et différents types de polarisation. Nous nous focaliserons sur le contrôle du premier mode de flexion de la structure.
\end{abstract}

Mots clés : Optimisation structurale / contrôle vibratoire / amortissement passif / shunt résistif / piézoélectricité

\begin{abstract}
Structural optimization of a piezoelectric patch shunted with a resistive circuit. The needs of encapsulation and autonomy of vibration control devices have opened the field of shunted piezoelectric materials. With their physical properties, piezoelectric element converts a ratio of vibration energy into electric energy. An electric circuit (shunt circuit) can be connected to the electrodes of the piezoelectric patch and thus modifies the dynamics of the whole system (structure + piezoelectric patches). In this paper, the studied control strategy is called passive vibration control because the shunt circuit is constituted by passive components as electric resistance or physical inductances. In literature, structural optimization of vibration control strategies is focused, in particular, on efficiency in terms of modal damping ratio. Energy flows between the different elements of the whole system are not considered. In this paper, a structural optimization criterion, taking into account modal damping ratio and dissipated energy, is developed. This criterion involves values easily performed with a finite element software. Let's note that one of the major modeling difficulties of this type of structures is related to the electric boundary conditions. A complete modeling or a representative modeling is necessary to compute precisely the
\end{abstract}

a Auteur pour correspondance : yann.meyer@supmeca.fr 
natural modes of the studied structure with a Dirichlet electric boundary condition and a Neumann electric boundary condition applied to the electrodes of the piezoelectric patches. The developed criterion is applied to a slender beam, on which a piezoelectric patch is bonded, for different mechanical boundary conditions and for different types of poling. The study is focused on the vibration control of the first bending natural mode of the structure.

Key words: Structural optimisation / vibration control / passive damping / resistive shunt / piezoelectricity

\section{Introduction}

La capacité des matériaux piézoélectriques à produire une réponse sous forme électrique lorsqu'ils sont mécaniquement contraints (effet direct) et inversement d'obtenir des mouvements de précision avec l'application d'un champ électrique (effet inverse) permet de les utiliser respectivement comme capteurs ou comme actionneurs, une fois placés sur des structures mécaniques flexibles. Les développements dans le domaine des structures dites « intelligentes », intégrant des capteurs et des actionneurs piézoélectriques, ont montré de grandes potentialités dans une large gamme d'applications tel que le diagnostic structural non destructif [1-3], le positionnement de précision [4-6], l'isolation vibratoire [7-12] ou l'amortissement de vibrations [13-17, 20,21].

Les besoins accrus d'encapsulation et d'autonomie des dispositifs de contrôle vibratoire ont ouvert le domaine des matériaux piézoelectriques «shuntés », c'està-dire connectés à un circuit électrique. Ces matériaux convertissent une partie de l'énergie vibratoire en énergie électrique et inversement. Par conséquent, il est possible en connectant à leurs bornes un circuit électrique de modifier leur dynamique propre et celle de leur structure porteuse. Ces processus ne requièrent ni capteur ni modèles numériques de la structure à contrôler. Cependant, pour tirer le meilleur parti de ces stratégies, une phase d'optimisation de l'ensemble du système est nécessaire.

L'optimisation des processus de stabilisation via des patchs piézoélectriques « shuntés » peuvent s'effectuer à deux niveaux : soit sur l'efficacité des impédances de contrôle, qu'elles soient actives, semi-actives ou passives, soit sur la forme et le placement des capteurs et des actionneurs.

Plusieurs techniques de shunt ont été développées et optimisées dans la littérature. L'idée fondamentale de toutes ces méthodes est de créer un circuit électrique équivalent, entre la capacité du patch piezoélectrique et le type d'impédance choisie, capable d'agir de manière optimale sur la structure porteuse afin de la stabiliser. Nous pouvons distinguer les shunts linéaires de type résistif [22], capacitif (positif ou négatif) [23-25], résonant monomodal $[22,26]$ ou résonant multimodal $[27,28]$ et les shunts non-linéaires à résistance commutée [29, 30], à inductance commutée [31,32], à raideur commutée [23] ou à source de tension commutée [32,33]. Les techniques de contrôle par shunt ont été largement étudiées pour stabiliser des structures mécaniques simples comme des poutres $[22,23,27,31]$ ou des plaques $[25,34]$ et, plus récemment, des systèmes plus complexes comme des rames de train [35], des systèmes de freinage [36] ou des ailerons d'appui de voiture de course [37].

Les performances des stratégies qu'elles soient actives, semi-actives ou passives dépendent fortement du nombre, de la taille, de la forme et du placement des couches piézoélectriques employées [38]. C'est sur ce point que, ces dernières années, les plus grands efforts se sont concentrés. Crawley et de Luis [18] ont émis l'idée d'un critère pour trouver le lieu optimal d'un actionneur piézoélectrique pour une poutre encastrée-libre. Baz et Poh [19] ont résolu le problème de l'optimisation, à la fois, du placement d'un actionneur possédant une taille pré-définie et de la tension optimale nécessaire au contrôle. L'utilisation de la méthode de contrôle dans l'espace modal indépendant modifié (Modified Independent Modal Space Control, MIMSC), où l'énergie des divers modes de la structure est estimée à partir de l'histoire des vibrations, est employée. Une fois cette valeur déterminée, les gains de rétroaction modaux optimaux du contrôleur sont calculés et seuls les modes possédant une haute énergie sont contrôlés. Crawley [18] a tenté de trouver le placement optimal des actionneurs piézoélectriques en déterminant le lieu de plus haute déformation moyenne sur la structure au cours du mouvement à contrôler. Devasia et al. [39] ont considéré le problème de l'optimisation de placement et de taille des actionneurs piézoélectriques distribués sur une poutre uniforme. Trois critères de performance sont étudiés :

- Cas de l'amortissement passif : la partie réelle des valeurs propres du système est maximisée en fonction des paramètres géométriques de la structure et des patchs de contrôle;

- Cas du régulateur quadratique linéaire (LQR) : une fonction de coût quadratique, construite avec la solution de l'équation algébrique de Riccati [40] et les conditions initiales du système, est maximisée;

- Cas de la grammienne de contrôlabilité : la contrôlabilité de tous les modes est maximisée.

Dhingra et Lee [41] ont étudié l'influence du placement des paires de capteurs et d'actionneurs et des gains de rétroaction sur la conception optimale des structures activement contrôlées. Pour le cas de l'optimisation du placement de l'actionneur, différentes fonctions coût et mesures d'efficacité ont également été employées. Quelques chercheurs $[42,43]$ ont proposé de maximiser le critère de contrôlabilité en utilisant une mesure de la matrice grammienne. Une fonction quadratique de coût tenant compte de l'erreur de mesure et de l'énergie de commande $[44,45]$ a également été considérée. Des recherches ont été 
menées [46] sur l'influence de l'épaisseur d'un piézoactionneur sur le contrôle des vibrations d'une poutre encastrée-libre en utilisant la théorie des poutres de EulerBernoulli. Meric et Saigal [47] ont dérivé les expressions de sensibilité de forme pour des structures linéaires avec des champs, mécanique et élastique, couplés. Les auteurs de [48] trouvent le placement optimal des paires d'actionneur-capteur piézoélectriques colocalisées sur une plaque mince encastrée à ces quatre bords. Cette optimisation est effectuée en déterminant le lieu de haute sensibilité de position pour chaque mode. Plusieurs chercheurs $[49,50]$ utilisent l'optimisation quadratique des performances pour trouver la position optimale des actionneurs et des capteurs piézoélectriques pour la suppression effective des vibrations structurales.

Comme nous pouvons le constater dans la littérature, la plus grande attention a été portée à l'optimisation de paires d'actionneur et de capteur. Leur placement, leur taille aussi bien que leur épaisseur sont considérés. Mais l'optimisation de systèmes de contrôle considérant conjointement le placement et la taille des patchs piézoélectriques et les impédances de contrôle c'est-à-dire de l'énergie nécessaire au contrôle n'a été que rarement étudiée, même dans le cas des stratégies actives $[51,52]$.

Dans cet article, nous proposons de développer un critère d'optimisation structural qui prend en compte la dissipation non seulement en termes d'amortissement induit par une technique de shunt résistif mais également en termes d'amplitude d'énergie dissipée.

L'article est divisé en trois parties. Nous développons, dans un premier temps, les aspects de modélisation comprenant les équations constitutives de la piézoélectricité ainsi que celles issues de la discrétisation par la méthode des éléments-finis. Ensuite, nous détaillons le critère d'optimisation structurale. Enfin, nous appliquons le critère au cas d'une poutre piézocomposite pour différentes conditions aux limites et pour différents modes de polarisation. Nous nous focaliserons sur le premier mode de flexion de la structure.

\section{Développement du modèle numérique}

\section{1 Équations d'équilibre}

Dans un domaine structural $\Omega_{0}$, le vecteur de déplacement $\boldsymbol{u}$ et le potentiel électrique $V$ sont solutions de l'équation d'équilibre et de la loi de Gauss en électrostatique.

$$
\begin{gathered}
\sigma_{i j, i}+\boldsymbol{f}_{j}=\rho \ddot{\boldsymbol{u}}_{j} \\
\boldsymbol{D}_{i, i}=\rho_{e}=0
\end{gathered}
$$

où $\sigma$ représente le tenseur des contraintes mécaniques $\left(\mathrm{N} . \mathrm{m}^{-2}\right), \boldsymbol{D}$ le déplacement électrique $\left(\mathrm{C} . \mathrm{m}^{-2}\right), \rho$ la masse volumique $\left(\mathrm{kg} . \mathrm{m}^{-3}\right), \rho_{\mathrm{e}}$ la densité volumique de charges $\left(\mathrm{C} . \mathrm{m}^{-3}\right)$ et $\boldsymbol{f}$ la densité volumique de forces extérieures s'appliquant sur la structure (N.m-3).
Le milieu n'étant pas infini, ces équations sont à compléter par des conditions aux limites.

Les conditions aux limites mécaniques associées au problème sont

$$
\left\{\begin{aligned}
\boldsymbol{u} & =\boldsymbol{u}_{0} \forall x \in \partial \Omega_{0}^{u} \\
\sigma_{i j} \cdot \boldsymbol{n}_{i} & =\bar{t}_{i} \forall x \in \partial \Omega_{0}^{T}
\end{aligned}\right.
$$

où $\partial \Omega_{0}^{u}$ et $\partial \Omega_{0}^{T}$ représentent respectivement les conditions de Dirichlet mécaniques et les conditions de Neumann mécaniques et $\boldsymbol{n}$ un vecteur normal à $\partial \Omega_{0}^{T}$.

Les conditions aux limites électriques associées au problème sont

$$
\left\{\begin{aligned}
V=0 & \forall x \in \partial \Omega_{0}^{V} \\
V & =V_{\mathrm{a}} \quad \forall x \in \partial \Omega_{\mathrm{a}}^{V} \\
{[\boldsymbol{D} \cdot \boldsymbol{n}] } & =0 \quad \forall x \in \partial \Omega_{0}^{q}
\end{aligned}\right.
$$

où $\partial \Omega_{0}^{V}, \partial \Omega_{\mathrm{a}}^{V}$ et $\partial \Omega_{0}^{q}$ représentent respectivement les conditions de Dirichlet électriques, les conditions de Dirichlet électriques des surfaces électriques d'entrée et de sortie du système et les conditions de Neumann électriques et $\boldsymbol{n}$ un vecteur normal à $\partial \Omega_{0}^{q}$.

\section{2 Équations de comportement}

À cause de leur symétrie, les tenseurs de contraintes $\boldsymbol{T}$ et de déformations $\boldsymbol{S}$ mécaniques peuvent être mis sous la forme de vecteurs contenant 6 termes indépendants en utilisant une notation matricielle condensée. Si les effets thermique et magnétique sont considérés négligeables, les équations couplées peuvent s'écrire, en notation condensée :

$$
\begin{aligned}
\boldsymbol{T}_{p} & =c_{p q}^{E} \cdot \boldsymbol{S}_{q}-e_{k p} \cdot \boldsymbol{E}_{k} \\
\boldsymbol{D}_{i} & =e_{i q} \cdot \boldsymbol{S}_{q}+\varepsilon_{i k}^{S} \cdot \boldsymbol{E}_{k} \\
\text { où } \quad \boldsymbol{E} & =-\nabla \cdot V
\end{aligned}
$$

$c^{E}, \varepsilon^{S}, e$ et $\boldsymbol{E}$ sont respectivement la matrice d'élasticité à champ électrique constant $\left(\mathrm{N} . \mathrm{m}^{-2}\right)$, la matrice de permittivité diélectrique à déformation constante $\left(\mathrm{F} \cdot \mathrm{m}^{-1}\right)$, la matrice de couplage électromécanique $\left(\mathrm{C} \cdot \mathrm{m}^{-2}\right)$ et le vecteur champ électrique $\left(\mathrm{V} \cdot \mathrm{m}^{-1}\right)$. La forme des matrices (7), (8) et (9) est caractéristique des céramiques PZT, polarisées suivant l'axe 3 .

$$
e_{k p}=\left[\begin{array}{cccccc}
0 & 0 & 0 & 0 & e_{15} & 0 \\
0 & 0 & 0 & e_{15} & 0 & 0 \\
e_{31} & e_{31} & e_{33} & 0 & 0 & 0
\end{array}\right]
$$




$$
\begin{gathered}
c_{p q}^{E}=\left[\begin{array}{cccccc}
c_{11} & c_{12} & c_{13} & 0 & 0 & 0 \\
c_{12} & c_{11} & c_{13} & 0 & 0 & 0 \\
c_{13} & c_{13} & c_{33} & 0 & 0 & 0 \\
0 & 0 & 0 & c_{44} & 0 & 0 \\
0 & 0 & 0 & 0 & c_{44} & 0 \\
0 & 0 & 0 & 0 & 0 & c_{66}
\end{array}\right] \\
\varepsilon_{i k}^{S}=\left[\begin{array}{cccc}
\varepsilon_{11} & 0 & 0 \\
0 & \varepsilon_{11} & 0 \\
0 & 0 & \varepsilon_{33}
\end{array}\right] .
\end{gathered}
$$

Il est à noter que les équations (5) et (6) sont applicables aux matériaux passifs en considérant $\varepsilon^{S}$ et $e$ comme des tenseurs nuls.

\subsection{Discrétisation par une méthode éléments-finis}

En combinant les relations (1) à (6), nous obtenons un système d'équations représentant le modèle de la structure étudiée. D'après [53], nous pouvons exprimer la formulation faible associée à ce modéle et ensuite appliquer la méthode des élèments-finis pour discrétiser le probléme. Nous obtenons alors la formulation éléments-finis matricielle (10).

$$
\begin{gathered}
\left(\begin{array}{ccc}
K_{\boldsymbol{u} \boldsymbol{u}} & K_{\boldsymbol{u} \boldsymbol{V}_{i}} & K_{\boldsymbol{u} \boldsymbol{V}_{p}} \\
K_{\boldsymbol{u} \boldsymbol{V}_{i}}^{t} & -K_{\boldsymbol{V}_{i} \boldsymbol{V}_{i}} & -K_{\boldsymbol{V}_{i} \boldsymbol{V}_{p}} \\
K_{\boldsymbol{u} \boldsymbol{V}_{p}}^{t} & -K_{\boldsymbol{V}_{i} \boldsymbol{V}_{p}}^{t}-K_{\boldsymbol{V}_{p} \boldsymbol{V}_{p}}
\end{array}\right)\left(\begin{array}{c}
\boldsymbol{u}_{n} \\
\boldsymbol{V}_{i} \\
\boldsymbol{V}_{p}
\end{array}\right) \\
+\left(\begin{array}{ccc}
M_{\boldsymbol{u} \boldsymbol{u}} & 0 & 0 \\
0 & 0 & 0 \\
0 & 0 & 0
\end{array}\right)\left(\begin{array}{c}
\ddot{\boldsymbol{u}}_{n} \\
\ddot{\boldsymbol{V}}_{i} \\
\ddot{\boldsymbol{V}}_{p}
\end{array}\right)=\left(\begin{array}{c}
\boldsymbol{F} \\
0 \\
\boldsymbol{Q}
\end{array}\right)
\end{gathered}
$$

où $\boldsymbol{V}_{i}, \boldsymbol{V}_{p}, \boldsymbol{u}_{n}, M_{\boldsymbol{u} \boldsymbol{u}}, K_{\boldsymbol{u} \boldsymbol{u}}, K_{\boldsymbol{u} \boldsymbol{V}_{*}}, K_{\boldsymbol{V}_{i} \boldsymbol{V}_{*}}$ et $K_{\boldsymbol{V}_{*} \boldsymbol{V}_{*}}$ sont respectivement le vecteur des potentiels nodaux interne à la structure, le vecteur des potentiels nodaux appliqués aux électrodes, le vecteur des déplacements nodaux, la matrice de masse, la matrice de raideur élastique, la matrice de raideur piézoélectrique et la matrice de raideur diélectrique. $\boldsymbol{F}$ et $\boldsymbol{Q}$ représentent respectivement le vecteur colonne des forces mécaniques appliquées aux nouds de la structure et le vecteur colonne des charges électriques mesurées en $\partial \Omega_{a}^{V}$.

Nous pouvons effectuer une condensation statique de type Guyan pour ne conserver que le vecteur colonne des électrodes, $\boldsymbol{V}_{p}$. De la deuxième ligne de l'équation (10), nous extrayons l'expression du potentiel interne à la structure.

$$
\boldsymbol{V}_{i}=K_{\boldsymbol{V}_{i} \boldsymbol{V}_{i}}^{-1}\left(K_{\boldsymbol{u} \boldsymbol{V}_{i}}^{t} \cdot \boldsymbol{u}_{n}-K_{\boldsymbol{V}_{i} \boldsymbol{V}_{p}} \cdot \boldsymbol{V}_{p}\right)
$$

En injectant (11) dans l'équation (10), nous obtenons le système matriciel (12).

$$
\left(\begin{array}{cc}
H_{\boldsymbol{u u}} & H_{\boldsymbol{u} \boldsymbol{V}_{p}} \\
H_{\boldsymbol{u} \boldsymbol{V}_{p}}^{t} & -H_{\boldsymbol{V}_{p} \boldsymbol{V}_{p}}
\end{array}\right)\left(\begin{array}{c}
\boldsymbol{u}_{n} \\
\boldsymbol{V}_{p}
\end{array}\right)+\left(\begin{array}{cc}
M_{\boldsymbol{u u}} & 0 \\
0 & 0
\end{array}\right)\left(\begin{array}{c}
\ddot{\boldsymbol{u}}_{n} \\
\ddot{\boldsymbol{V}}_{p}
\end{array}\right)=\left(\begin{array}{c}
\boldsymbol{F} \\
\boldsymbol{Q}
\end{array}\right)
$$

avec

$$
\left\{\begin{array}{l}
H_{\boldsymbol{u} \boldsymbol{u}}=K_{\boldsymbol{u} \boldsymbol{u}}+K_{\boldsymbol{u} \boldsymbol{V}_{i}} K_{\boldsymbol{V}_{i} \boldsymbol{V}_{i}}^{-1} K_{\boldsymbol{u} \boldsymbol{V}_{i}}^{t} \\
H_{\boldsymbol{u} \boldsymbol{V}_{p}}=K_{\boldsymbol{u} \boldsymbol{V}_{p}}-K_{\boldsymbol{u} \boldsymbol{V}_{p}} K_{\boldsymbol{V}_{i} \boldsymbol{V}_{i}}^{-1} K_{\boldsymbol{V}_{i} \boldsymbol{V}_{p}}^{t} \\
H_{\boldsymbol{V}_{p} \boldsymbol{V}_{p}}=K_{\boldsymbol{V}_{p} \boldsymbol{V}_{p}}^{t}+K_{\boldsymbol{V}_{i} \boldsymbol{V}_{p}}^{t} K_{\boldsymbol{V}_{i} \boldsymbol{V}_{i}}^{-1} K_{\boldsymbol{V}_{i} \boldsymbol{V}_{p}}
\end{array}\right.
$$

Il est nécessaire d'appliquer la condition d'équipotentialité aux électrodes. L'équation (13) traduit le fait que le potentiel des électrodes possède une valeur identique quel que soit le point considéré sur l'électrode.

$$
\boldsymbol{V}_{p}=I_{v} \cdot \boldsymbol{V}_{a}
$$

avec $I_{v}$, une matrice de localisation des électrodes composée de 1 et de 0 .

Nous appliquons l'équation (13) au système (12).

$$
\left(\begin{array}{cc}
H_{\boldsymbol{u} \boldsymbol{u}} & H_{\boldsymbol{u} \boldsymbol{V}_{a}} \\
H_{\boldsymbol{u} \boldsymbol{V}_{a}}^{t} & -H_{\boldsymbol{V}_{a} \boldsymbol{V}_{a}}
\end{array}\right)\left(\begin{array}{c}
\boldsymbol{u}_{n} \\
\boldsymbol{V}_{a}
\end{array}\right)+\left(\begin{array}{cc}
M_{\boldsymbol{u} \boldsymbol{u}} & 0 \\
0 & 0
\end{array}\right)\left(\begin{array}{c}
\ddot{\boldsymbol{u}}_{n} \\
\ddot{\boldsymbol{V}}_{a}
\end{array}\right)=\left(\begin{array}{c}
\boldsymbol{F} \\
\boldsymbol{q}_{a}
\end{array}\right)
$$

avec

$$
\left\{\begin{array}{l}
H_{\boldsymbol{u} \boldsymbol{V}_{a}}=H_{\boldsymbol{u} \boldsymbol{V}_{p}} \cdot I_{v} \\
H_{\boldsymbol{V}_{a} \boldsymbol{V}_{a}}=I_{v}^{t} \cdot H_{\boldsymbol{V}_{p} \boldsymbol{V}_{p}} \cdot I_{v} \\
\boldsymbol{q}_{a}=I_{v}^{t} \cdot \boldsymbol{Q}
\end{array}\right.
$$

$H_{\boldsymbol{V}_{a} \boldsymbol{V}_{a}}$ est une matrice diagonale contenant les capacités des patchs piézoélectriques employés et $\boldsymbol{q}_{a}$ les charges électriques issues de ces patchs.

Nous pouvons également écrire le système d'équations (14) sous la forme suivante (on pose $\boldsymbol{F}=0)$ :

$$
\begin{gathered}
M_{\boldsymbol{u u}} \cdot \ddot{\boldsymbol{u}}_{n}+\left(H_{\boldsymbol{u u}}+\Delta H_{\boldsymbol{u u}}\right) \cdot \boldsymbol{u}_{n}=H_{\boldsymbol{u} \boldsymbol{V}_{a}} \cdot H_{\boldsymbol{V}_{a} \boldsymbol{V}_{a}}^{-1} \cdot \boldsymbol{q}_{a} \\
H_{\boldsymbol{V}_{a} \boldsymbol{V}_{a}}^{-1} \cdot H_{\boldsymbol{u} \boldsymbol{V}_{a}}^{t} \cdot \boldsymbol{u}_{n}-H_{\boldsymbol{V}_{a} \boldsymbol{V}_{a}}^{-1} \cdot \boldsymbol{q}_{a}=\boldsymbol{V}_{a}
\end{gathered}
$$

où

$$
\Delta H_{\boldsymbol{u} \boldsymbol{u}}=H_{\boldsymbol{u} \boldsymbol{V}_{a}} \cdot H_{\boldsymbol{V}_{a} \boldsymbol{V}_{a}}^{-1} \cdot H_{\boldsymbol{u} \boldsymbol{V}_{a}}^{t}
$$

\subsection{Modes de vibration de la structure}

Dans le cas d'un shunt de type résistif, $\boldsymbol{q}_{a}$ et $\boldsymbol{V}_{a}$ sont liés par la loi d'Ohm. Nous pouvons exprimer cette relation dans le domaine de Laplace avec $s$ la variable de Laplace et $R$ la matrice diagonale contenant les valeurs de résistances de shunt.

$$
\boldsymbol{V}_{a}=R s \boldsymbol{q}_{a}
$$




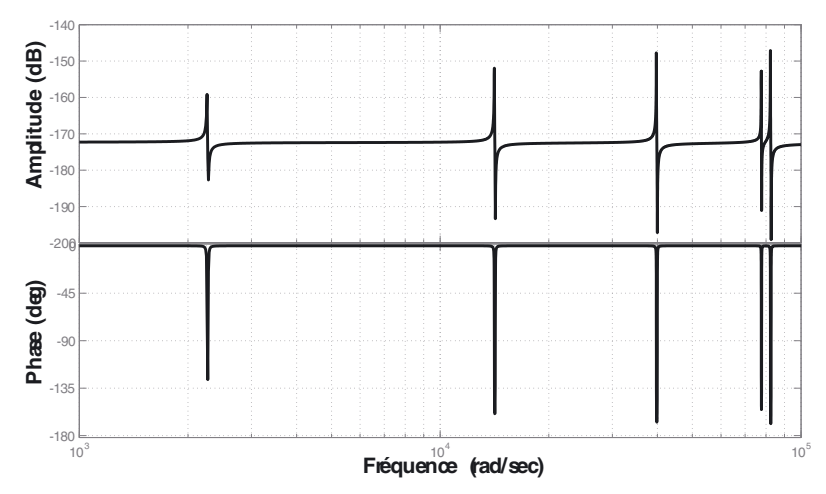

Fig. 1. Diagramme de Bode typique de l'admittance électrique d'une structure piézoélectrique.

Nous injectons la relation (17) dans les équations (15) et (16) afin d'éliminer le potentiel électrique. Nous posons $G=\left(R H_{\boldsymbol{V}_{a} \boldsymbol{V}_{a}}\right)^{-1}$. Nous obtenons les relations suivantes :

$$
\begin{aligned}
& M_{\boldsymbol{u} \boldsymbol{u}} s^{2} \cdot \boldsymbol{u}_{n}+\left(H_{\boldsymbol{u} \boldsymbol{u}}+\Delta H_{\boldsymbol{u} \boldsymbol{u}}-\right. \\
& \left.H_{\boldsymbol{u} \boldsymbol{V}_{a}} \cdot H_{\boldsymbol{V}_{a} \boldsymbol{V}_{a}}^{-1}(s I+G)^{-1} G H_{\boldsymbol{u} \boldsymbol{V}_{a}}^{t}\right) \cdot \boldsymbol{u}_{n}=0
\end{aligned}
$$

$$
\boldsymbol{q}_{a}=(s I+G)^{-1} G H_{\boldsymbol{u} \boldsymbol{V}_{a}}^{t} \cdot \boldsymbol{u}_{n}
$$

avec $I$ la matrice identité de mêmes dimensions que la matrice $H_{\boldsymbol{V}_{a} \boldsymbol{V}_{a}}$.

Si nous considérons un seul patch piézoélectrique alors $H_{\boldsymbol{V}_{a} \boldsymbol{V}_{a}}$ est un scalaire. Si $R \longrightarrow 0$ alors $G \longrightarrow \infty$. L'équation de mouvement (18) de la structure mécanique s'écrit alors :

$$
M_{\boldsymbol{u} \boldsymbol{u}} s^{2} \cdot \boldsymbol{u}_{n}+H_{\boldsymbol{u u}} \cdot \boldsymbol{u}_{n}=0
$$

La relation (20) correspond au mode de vibration de la structure à $V_{a}=0$ c'est-à-dire en court circuit électrique. Nous avons accès aux modes de vibrations purement mécaniques, appelés résonances de la structure.

Si $R \longrightarrow \infty$ alors $G \longrightarrow 0$. L'équation de mouvement (18) de la structure mécanique s'écrit alors :

$$
M_{\boldsymbol{u} u} s^{2} \cdot \boldsymbol{u}_{n}+\left(H_{\boldsymbol{u u}}+\Delta H_{\boldsymbol{u} \boldsymbol{u}}\right) \cdot \boldsymbol{u}_{n}=0
$$

La relation (21) correspond au mode de vibration de la structure à $q_{a}=0$, c'est-à-dire en circuit ouvert. Nous avons alors accès aux modes de vibrations de couplage piézoélectrique, appelés anti-résonances de la structure.

Sur la figure 1, nous observons une courbe d'admittance typique d'une structure piézoélectrique avec une alternance résonance-antirésonance.

\section{Critère d'optimisation}

\section{1 Étude de sensibilité}

Les relations (18) et (19) sont réécrites sous la forme du système (22) dans le domaine de Laplace.

$$
\left[\begin{array}{cc}
M_{\boldsymbol{u} \boldsymbol{u}} s^{2}+H_{\boldsymbol{u} \boldsymbol{u}}+\Delta H_{\boldsymbol{u u}} & -H_{\boldsymbol{u} \boldsymbol{V}_{a}} H_{\boldsymbol{V}_{a} \boldsymbol{V}_{a}}^{-1} \\
-G H_{\boldsymbol{u} \boldsymbol{V}_{a}}^{t} & (s I+G)
\end{array}\right]\left[\begin{array}{c}
\boldsymbol{u} \\
\boldsymbol{q}_{a}
\end{array}\right]=\left[\begin{array}{l}
0 \\
0
\end{array}\right]
$$

Nous projetons le déplacement $\boldsymbol{u}$ sur la base des modes propres $\left\{\boldsymbol{\phi}_{i}, \Omega_{i}\right\}_{i=1 \ldots N}$ en circuit ouvert $\left(\boldsymbol{q}_{a}=0\right)$ avec $\phi_{i}$, le vecteur de déformée propre du mode $i$ et $\Omega_{i}$, la valeur propre du mode $i$. Les vecteurs $\phi_{i}$ sont normalisés par rapport à la matrice de masse. Nous obtenons, en convention d'Einstein, l'expression (23).

$$
\boldsymbol{u}=\phi_{i} \eta_{i}
$$

On suppose que tous les patchs piézoélectriques sont de mêmes dimensions donc possèdent tous la même capacité $H_{\boldsymbol{V}_{a} \boldsymbol{V}_{a}}$. En injectant la relation (23) dans le système (22), nous obtenons l'équation matricielle (24).

$$
\left[\begin{array}{cc}
I_{N} s^{2}+\Omega^{2} & -\phi^{t} H_{\boldsymbol{u} \boldsymbol{V}_{a}} H_{\boldsymbol{V}_{a} \boldsymbol{V}_{a}}^{-1} \\
-G H_{\boldsymbol{u} \boldsymbol{V}_{a}}^{t} \phi & (s+G) I
\end{array}\right]\left[\begin{array}{l}
\boldsymbol{\eta} \\
\boldsymbol{q}_{a}
\end{array}\right]=\left[\begin{array}{l}
0 \\
0
\end{array}\right]
$$

avec $I_{N}$ la matrice identité de dimensions $N, \Omega$ la matrice diagonale contenant les pulsations $\Omega_{i}, \phi$ la matrice contenant les déformées modales $\phi_{i}$ et $\boldsymbol{\eta}$ le vecteur colonne contenant les contributions modales $\eta_{i}$.

Séparons les variables de l'équation (24).

$$
\left[s^{3} I_{N}+s^{2} G I_{N}+s \Omega^{2}+\left(\Omega^{2}-\phi^{t} H_{\boldsymbol{u} \boldsymbol{V}_{a}} H_{\boldsymbol{V}_{a} \boldsymbol{V}_{a}}^{-1} H_{\boldsymbol{u} \boldsymbol{V}_{a}}^{t} \phi\right) G\right] \boldsymbol{\eta}=0
$$

$\left[-G H_{\boldsymbol{u} \boldsymbol{V}_{a}}^{t} \phi\left(s^{2} I_{N}+\Omega^{2}\right)^{-1} \phi^{t} H_{\boldsymbol{u} \boldsymbol{V}_{a}} H_{\boldsymbol{V}_{a} \boldsymbol{V}_{a}}^{-1}+(s+G) I\right] \boldsymbol{q}_{a}=0$

Si nous considérons les pôles en circuit fermé, nous avons l'équation (27).

$$
\omega^{2}=\Omega^{2}-\operatorname{diag}\left(\phi^{t} \Delta H_{\boldsymbol{u} \boldsymbol{u}} \phi\right)
$$

En considérant la relation (27), l'équation (25) s'écrit sous la forme (28)

$$
\left[s^{3} I_{N}+s^{2} G I_{N}+s \Omega^{2}+\omega^{2} G-G R_{e s u}\right] \boldsymbol{\eta}=0
$$

avec $R_{\text {esu }}$ une matrice à diagonale nulle ne comportant que les termes extradiagonaux de la matrice $\phi^{t} \Delta H_{\boldsymbol{u} \boldsymbol{u}} \phi$. La matrice $R_{\text {esu }}$ peut être considérée comme négligeable pour des faibles valeurs de $G$ [52].

L'étude porte sur la sensibilité des pôles liés à la structure, $s_{m}$, et ceux liés au shunt par rapport à la valeur de la résistance de shunt, $s_{\mathrm{g}}$. Il nous faut déterminer les variations de ces pôles en fonction des différents paramètres du système. 
Nous considérons les pôles initiaux décrits par la famille (29).

$$
\left\{s_{m}^{0}, s_{\mathrm{g}}^{0}\right\}=\left\{\left\{ \pm j \Omega_{i}\right\}_{i=1 \ldots N},\{0\}_{k=1 \ldots n}\right\}
$$

Les pôles liés à la structure mécanique sont solutions du déterminant de l'équation (28). La sensibilité des pôles mécaniques par rapport à $G$, autour de la valeur 0 , est donnée par la relation (30).

$$
\left(\frac{\partial s}{\partial G}\right)_{s=s_{m}^{0}, G=0}=\left(\frac{\frac{\partial H_{1}(s, G)}{\partial G}}{\frac{\partial H_{1}(s, G)}{\partial s}}\right)_{s=s_{m}^{0}, G=0}
$$

avec $H_{1}(s, G)$ le déterminant de l'équation (28).

D'après [52], l'expression de l'évolution des pôles mécaniques du système contrôlé, autour $G=0$ et au premier ordre en $G$, est exprimée par l'équation (31).

$$
\begin{gathered}
s_{m i}=-G \frac{\left(\Omega_{i}^{2}-\omega_{i}^{2}\right)}{2 \Omega_{i}^{2}} \pm j \Omega_{i} \\
\Longleftrightarrow s_{m i}=-\left(R H_{\boldsymbol{V}_{a} \boldsymbol{V}_{a}}\right)^{-1} \frac{\left(\Omega_{i}^{2}-\omega_{i}^{2}\right)}{2 \Omega_{i}^{2}} \pm j \Omega_{i}
\end{gathered}
$$

Pour stabiliser au mieux la structure, c'est-à-dire pour obtenir le meilleur facteur d'amortissement, il faut augmenter la distance entre la fréquence propre du mode $i$ en circuit ouvert, $\Omega_{i}$, et la fréquence propre du mode $i$ en circuit fermé, $\omega_{i}$.

Les pôles liés au shunt sont les solutions du déterminant de la relation (26). La sensibilité des pôles liés au shunt par rapport à $G$, autour de la valeur 0 , est exprimée par la relation (32).

$$
\begin{aligned}
H_{2}(s= & 0, G)=0 \\
& =\operatorname{det}\left(-G H_{\boldsymbol{u} \boldsymbol{V}_{a}}^{t} \phi\left(\Omega^{2}\right)^{-1} \phi^{t} H_{\boldsymbol{u} \boldsymbol{V}_{a}} H_{\boldsymbol{V}_{a} \boldsymbol{V}_{a}}^{-1}+(s+G) I\right)
\end{aligned}
$$

avec $H_{2}(s, G)$ le déterminant de l'équation (26).

Grâce à l'orthogonalité des modes propres et à la normalisation des vecteurs propres par rapport à la masse, nous obtenons $\phi\left(\Omega^{2}\right)^{-1} \phi^{t}=K^{-1} . K^{-1}$ correspond à la matrice de compliance de la structure. L'équation (32) peut se mettre sous la forme de la relation (33).

$$
\operatorname{det}\left(-G H_{\boldsymbol{u} \boldsymbol{V}_{a}}^{t} K^{-1} H_{\boldsymbol{u} \boldsymbol{V}_{a}} H_{\boldsymbol{V}_{a} \boldsymbol{V}_{a}}^{-1}+(s+G) I\right)=0
$$

La matrice carrée $H_{\boldsymbol{u} \boldsymbol{V}_{a}}^{t} K^{-1} H_{\boldsymbol{u} \boldsymbol{V}_{a}}$ correspond au complément dual de Schur associé à la distribution des patchs sur la structure mécanique. Nous projetons l'équation (33) sur la base des modes propres $\left\{\boldsymbol{\psi}_{i}, L_{i}\right\}_{i=1 \ldots N}$ associée à la matrice $H_{\boldsymbol{u} \boldsymbol{V}_{a}}^{t} K^{-1} H_{\boldsymbol{u} \boldsymbol{V}_{a}}$ de dimensions $n \times n$ avec $\boldsymbol{\psi}_{i}$ le vecteur des déformées propres du mode $i$ et $L_{i}$ la valeur propre du mode $i$. Les vecteurs $\boldsymbol{\psi}_{i}$ sont normalisés pour obtenir $\psi^{t} \psi=I$. Les pôles, solutions de l'équation (33), sont également solutions de la relation (34).

$$
\operatorname{det}\left(-G L H_{\boldsymbol{V}_{a} \boldsymbol{V}_{a}}^{-1}+(s+G) I\right)=0
$$

avec $L$ la matrice diagonale contenant les valeurs propres $L_{i}$.

Nous obtenons facilement la relation (35).

$$
\left(\frac{\partial s}{\partial G}\right)_{s=s_{g}^{0}, G=0}=-G L H_{\boldsymbol{V}_{a} \boldsymbol{V}_{a}}^{-1}+I
$$

On en déduit alors l'expression (36) de l'évolution des pôles de shunt, autour $G=0$ et au premier ordre en $G$.

$$
s_{g k}=G\left(\left(H_{\boldsymbol{V}_{a} \boldsymbol{V}_{a}}^{-1}\right)_{k} L_{k}-1\right)
$$

Par définition d'un shunt résistif, le système est stable. Les pôles de shunt restent donc négatifs. Pour limiter le niveau de la charge $q_{a}$, il est nécessaire de maximiser $\left|s_{g k}\right|$.

\subsection{Obtention du critère}

D'après l'équation (27) et à couplage constant, plus la raideur $\Delta H_{\boldsymbol{u} u}$, induite par les conditions électriques, est élevée, plus les pôles $\omega_{i}$ et $\Omega_{i}$ sont éloignés. Donc, plus la capacité du patch est faible, plus la distance entre les pôles $\omega_{i}$ et $\Omega_{i}$ est grande. Le facteur d'amortissement et la valeur de résitance en sont d'autant plus élevés. Cet effet est limité par les effets de raideur ajoutée par la structure mécanique du patch.

Issu de la relation $(36),\left(H_{\boldsymbol{V}_{a} \boldsymbol{V}_{a}}^{-1}\right)_{k} L_{k}-1=$ $\frac{L_{k}-\left(H_{\left.\boldsymbol{V}_{a} \boldsymbol{V}_{a}\right)_{k}}\right.}{\left(H_{\left.\boldsymbol{V}_{a} \boldsymbol{V}_{a}\right)_{k}}\right.}$ représente le ratio apparent entre la capacité équivalente de la structure mécanique au niveau de la localisation du patch $k$ et celle du patch $k$. Par conséquent, à couplage constant, plus le patch $k$ possède une capacité élevée par rapport à la capacité équivalente de la structure mécanique, plus faible est la valeur de la résistance de shunt mais plus limitées sont les performances en termes d'amortissement. Il est donc nécessaire de trouver un compromis entre performances et dissipation d'énergie.

Sur le modèle du critère développé en [52], nous proposons le critère (37).

$$
\begin{aligned}
J_{i} & =\frac{\left(\Omega_{i}^{2}-\omega_{i}^{2}\right)}{2 \Omega_{i}^{2}} \min _{k=1 \ldots n}\left(\left(H_{\boldsymbol{V}_{a} \boldsymbol{V}_{a}}^{-1}\right)_{k} L_{k}-1\right) \\
\text { pour } \quad i & =1 \ldots N
\end{aligned}
$$

Les variables de conception associées au critère sont les pulsations $\Omega_{i}$ à circuit ouvert, les pulsations $\omega_{i}$ à circuit fermé, la capacité des patchs $H_{\boldsymbol{V}_{a} \boldsymbol{V}_{a}}$ de contrôle et les valeurs propres $L_{k}$ associées à la matrice $H_{\boldsymbol{u} \boldsymbol{V}_{a}}^{t} K^{-1} H_{\boldsymbol{u} \boldsymbol{V}_{a}} \cdot \frac{\left(\Omega_{i}^{2}-\omega_{i}^{2}\right)}{2 \Omega_{i}^{2}}$ évalue l'amortissement modal de la structure complète. $\min _{k=1 \ldots n}\left(\left(H_{\boldsymbol{V}_{a} \boldsymbol{V}_{a}}^{-1}\right)_{k} L_{k}-1\right)$ donne la plus faible valeur de résistance nécessaire pour contrôler la structure.

\section{Simulations numériques : cas d'une poutre piézocomposite}

Pour évaluer les performances du critère (37), nous l'appliquons au cas d'une poutre vibrante en flexion, sur 


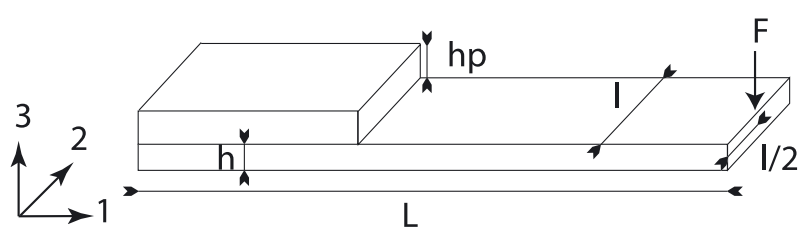

Fig. 2. Dimensions et mode de sollicitation de la poutre étudiée.

Tableau 1. Paramètres dimensionnels de la poutre et du patch.

\begin{tabular}{ccc}
\hline Paramètre & Valeur & unités \\
\hline$L$ & 100 & $\mathrm{~mm}$ \\
$l$ & 10 & $\mathrm{~mm}$ \\
$h$ & 4 & $\mathrm{~mm}$ \\
$h_{p}$ & 1 & $\mathrm{~mm}$ \\
\hline
\end{tabular}

Tableau 2. Paramètres du matériau constitutif de la poutre.

\begin{tabular}{ccc}
\hline Paramètre & Valeur & unités \\
\hline$E$ & 200 & GPa \\
$\nu$ & 0,33 & - \\
$\rho$ & 7850 & kg.m \\
\hline
\end{tabular}

Tableau 3. Paramètres du matériau constitutif du patch PZT-5H.

\begin{tabular}{ccc}
\hline Paramètre & Valeur & unités \\
\hline$\rho$ & 7500 & $\mathrm{~kg} . \mathrm{m}^{-3}$ \\
$e_{31}$ & $-6,62$ & $\mathrm{C} . \mathrm{m}^{-2}$ \\
$e_{33}$ & 23,24 & $\mathrm{C} . \mathrm{m}^{-2}$ \\
$e_{15}$ & 17,03 & $\mathrm{C} . \mathrm{m}^{-2}$ \\
$c_{11}$ & 127,2 & $\mathrm{GPa}$ \\
$c_{12}$ & 80,2 & $\mathrm{GPa}$ \\
$c_{13}$ & 84,6 & $\mathrm{GPa}$ \\
$c_{33}$ & 117,4 & $\mathrm{GPa}$ \\
$c_{44}$ & 22,9 & $\mathrm{GPa}$ \\
$c_{66}$ & 23,4 & $\mathrm{GPa}$ \\
$\varepsilon_{11}$ & 150,91 & $10^{-11} \mathrm{~F} . \mathrm{m}^{-1}$ \\
$\varepsilon_{33}$ & 126,93 & $10^{-11} \mathrm{~F} . \mathrm{m}^{-1}$ \\
\hline
\end{tabular}

laquelle un patch piézoélectrique est collé. L'optimisation porte sur la longueur du patch. Nous étudions différents modes de polarisation et différentes conditions aux limites.

\subsection{Description de la structure}

La structure mécanique est une poutre en acier sur laquelle un patch piézoélectrique est collé, comme présenté en figure 2. Les dimensions et les paramètres des matériaux sont donnés dans les tableaux 1 et 2 . Il est à noter que la longueur du patch constitue la donnée à optimiser. Pour solliciter la poutre, nous appliquons une force $F$ sur le bord non-encastré au milieu de la largeur de la poutre, comme décrit en figure 2 .

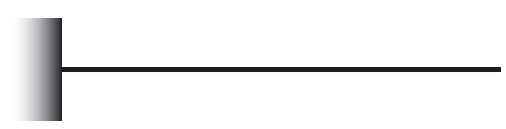

Fig. 3. Condition aux limites 1 : poutre encastrée-libre.

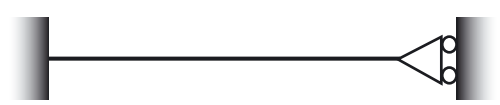

Fig. 4. Condition aux limites 2 : poutre encastrée-guidée.

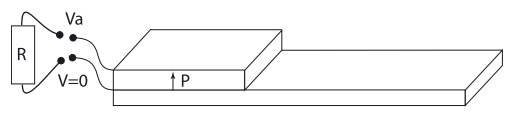

Fig. 5. Polarisation du patch en mode d'extension.

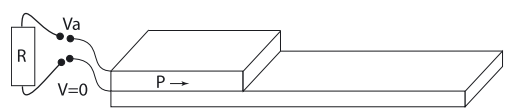

Fig. 6. Polarisation du patch en mode de cisaillement.

Nous considérons deux types de conditions aux limites :

- La condition 1, donnée en figure 3 où la poutre est encastrée-libre,

- la condition 2, donnée en figure 4 où la poutre est encastrée-guidée.

Nous considérons également deux types de polarisation correspondant à deux types d'actionnement :

- La polarisation 1 (ou actionnement en extension), donnée en figure 5 où l'axe de polarisation du patch piézoélectrique est colinéaire à l'axe du champ électrique.

- la polarisation 2 (ou actionnement en cisaillement), donnée en figure 6 où l'axe de polarisation du patch piézoélectrique est perpendiculaire à l'axe du champ électrique et parallèle à l'axe longitudinal de la poutre. Les lois de comportement correspondantes s'obtiennent en inter-changeant les directions 3 et 1 .

\subsection{Modéle numérique de la structure}

Les équations multiphysiques du système sont discrétisées par une méthode éléments finis en utilisant le logiciel Comsol Multiphysics ${ }^{\circledR}$. La structure est maillée en utilisant des éléments hexaèdriques de type Lagrange quadratique avec 8 points de Gauss par élément. Le maillage possède 10385 degrés de liberté pour 336 éléments. Comme illustré en figure 7 , l'épaisseur du patch contient 4 éléments et l'épaisseur de la poutre 3 éléments. Pour réduire la taille des matrices, une méthode de condensation dynamique est utilisée [54]. Il s'agit d'une méthode de Craig et Bampton modifiée dans laquelle la charge statique du patch est incluse dans la base de condensation. 


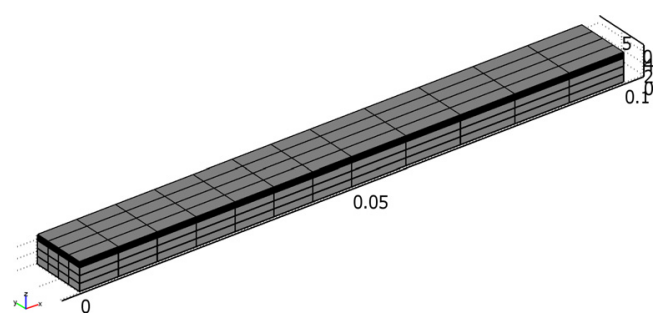

Fig. 7. Maillage de la poutre piézocomposite.

\section{Résultats numériques}

Pour cette étude numérique, nous considérons le premier mode de flexion de la poutre en dynamique pour les deux conditions aux limites définies en figures 3 et 4 et pour les deux modes d'actionnement. Pour l'ensemble de l'étude, nous supposons un facteur d'amortissement initial de $0,1 \%$. Celui-ci est pris en compte par addition aux performances obtenues grâce au critère. La plage d'optimisation de la longueur du patch s'étend de $10 \%$ à $90 \%$ de la longueur totale de la poutre soit de $10 \mathrm{~mm}$ à $90 \mathrm{~mm}$.

\subsection{Mode d'extension}

La figure 5 présente le mode d'actionnement étudié. Il s'agit d'un mode d'extension-compression du patch qui permet de faire fléchir la poutre lorsque la plaquette piézoélectrique est collée sur l'une de ces faces.

\subsubsection{Poutre encastrée-libre}

Dans un premier temps, nous optimisons grâce au critère la longueur du patch piézoélectrique. À titre de comparaison, nous optimisons également la longueur du patch d'un point de vue «amortissement modal $»$ en considérant uniquement le terme $\frac{\left(\Omega_{i}^{2}-\omega_{i}^{2}\right)}{2 \Omega_{i}^{2}}$. C'est ce que nous nommons «performances brutes ». Sur la figure 8 , nous superposons les performances brutes et le critère en fonction de la longueur du patch. Il apparaît clairement une différence entre les optimaux des deux critères. La longueur optimale pour les performances brutes est obtenue à $55 \%$ de la longueur totale de la poutre soit $55 \mathrm{~mm}$ et à $70 \%$ de la longueur totale de la poutre soit $70 \mathrm{~mm}$ pour l'optimisation grâce au critère.

Dans un deuxième temps, nous mettons en place la résistance de shunt pour contrôler la structure. Le tableau 4 donne un comparatif de résistances à valeur constante d'amortissement du mode 1 pour les deux optimisations étudiées. La structure optimisée montre un gain sur la valeur de résistances donc sur l'énergie de contrôle.

De la même manière, le tableau 5 donne un comparatif de performances à valeur de résistance constante pour les deux optimisations étudiées. Nous constatons une augmentation des performances entre les deux processus pour une résitance égale.

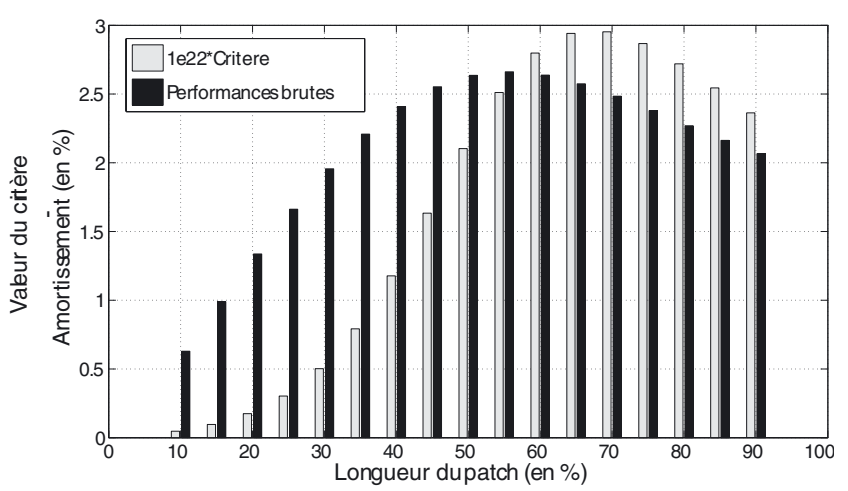

Fig. 8. Comparaison entre les performances brutes et le critère en fonction de la longueur du patch, dans le cas de l'actionnement par extension et des conditions aux limites de type encastrée-libre au mode 1.

Tableau 4. Comparaison entre les performances brutes et les performances optimisées à amortissement constant dans le cas de l'actionnement par extension et des conditions aux limites de type encastrée-libre au mode 1.

\begin{tabular}{ccc}
\hline $\begin{array}{c}\text { Amortissement } \\
(\text { en } \%)\end{array}$ & $\begin{array}{c}\text { Résistance brute } \\
(\mathrm{k} \Omega)\end{array}$ & $\begin{array}{c}\text { Résistance } \\
\text { optimisée }(\mathrm{k} \Omega)\end{array}$ \\
\hline 1,25 & 3,11 & 2,69 \\
1,5 & 5,93 & 5,07 \\
1,75 & 9 & 7,96 \\
2 & 13 & 11,8 \\
2,2 & 18,2 & 17,8 \\
\hline
\end{tabular}

Tableau 5. Comparaison entre les performances brutes et les performances optimisées à valeur de résistance constante dans le cas de l'actionnement par extension et des conditions aux limites de type encastrée-libre au mode 1.

\begin{tabular}{ccc}
\hline $\begin{array}{c}\text { Résitance } \\
(\mathrm{k} \Omega)\end{array}$ & $\begin{array}{c}\text { Amortissement brut } \\
(\text { en \%) }\end{array}$ & $\begin{array}{c}\text { Amortissement } \\
\text { optimisé (en \%) }\end{array}$ \\
\hline 5 & 1,42 & 1,49 \\
10 & 1,82 & 1,9 \\
13 & 2 & 2,06 \\
15 & 2,09 & 2,13 \\
\hline
\end{tabular}

\subsubsection{Poutre encastrée-guidée}

Sur la figure 9, les courbes de performances brutes et du critère en fonction de la longueur du patch sont superposées. La longueur optimale pour les performances brutes est obtenue à $40 \%$ de la longueur totale de la poutre soit $40 \mathrm{~mm}$ et à $55 \%$ de la longueur totale de la poutre soit $55 \mathrm{~mm}$ pour l'optimisation grâce au critère.

Nous mettons en place la résistance de shunt pour contrôler la structure. Le tableau 6 donne un comparatif de résistances à valeur constante d'amortissement du mode 1 pour les deux optimisations étudiées. La structure optimisée montre un gain sur la valeur de la résistance de shunt donc sur l'énergie de contrôle. 


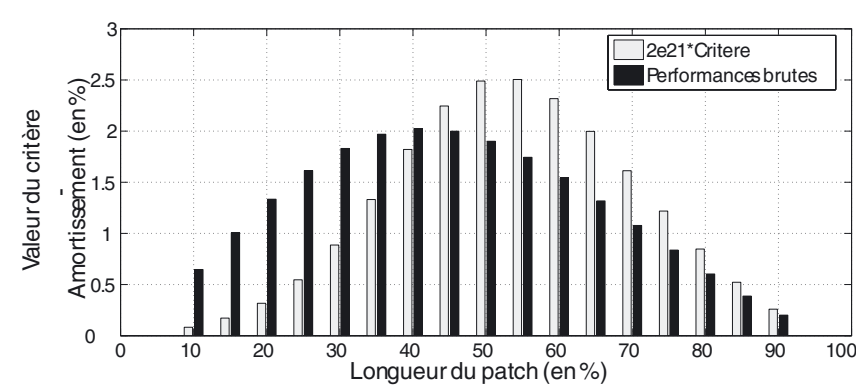

Fig. 9. Comparaison entre les performances brutes et le critère en fonction de la longueur du patch, dans le cas de l'actionnement par extension et des conditions aux limites de type encastrée-guidée au mode 1.

Tableau 6. Comparaison entre les performances brutes et les performances optimisées à amortissement constant dans le cas de l'actionnement par extension et des conditions aux limites de type encastrée-guidée au mode 1.

\begin{tabular}{ccc}
\hline $\begin{array}{c}\text { Amortissement } \\
(\text { en } \%)\end{array}$ & $\begin{array}{c}\text { Résistance brute } \\
(\mathrm{k} \Omega)\end{array}$ & $\begin{array}{c}\text { Résistance } \\
\text { optimisée }(\mathrm{k} \Omega)\end{array}$ \\
\hline 1,1 & 1,83 & 1,57 \\
1,25 & 3,85 & 3,34 \\
1,5 & 7,54 & 6,72 \\
1,75 & 12,7 & 12,5 \\
1,8 & 14 & 14,9 \\
\hline
\end{tabular}

Tableau 7. Comparaison entre les performances brutes et les performances optimisées à valeur de résistance constante dans le cas de l'actionnement par extension et des conditions aux limites de type encastrée-guidée au mode 1.

\begin{tabular}{ccc}
\hline $\begin{array}{c}\text { Résitance } \\
(\mathrm{k} \Omega)\end{array}$ & $\begin{array}{c}\text { Amortissement brut } \\
(\text { en } \%)\end{array}$ & $\begin{array}{c}\text { Amortissement } \\
\text { optimisé (en \%) }\end{array}$ \\
\hline 1,5 & 1,07 & 1,1 \\
3 & 1,19 & 1,22 \\
5 & 1,33 & 1,38 \\
7,5 & 1,49 & 1,54 \\
10 & 1,64 & 1,67 \\
\hline
\end{tabular}

Le tableau 7 donne un comparatif de performances à valeur de résistance constante pour les deux optimisations étudiées. Il apparaît que la stratégie optimisée via le critère (37) requiert une résistance moins importante que, dans le cas de l'optimisation modale, à performance égale.

\subsection{Mode de cisaillement}

La figure 6 présente le mode d'actionnement considéré. Il s'agit d'un mode de cisaillement du patch qui permet de faire fléchir la poutre par rotation des sections lorsque la plaquette piézoélectrique est collée sur l'une des faces.

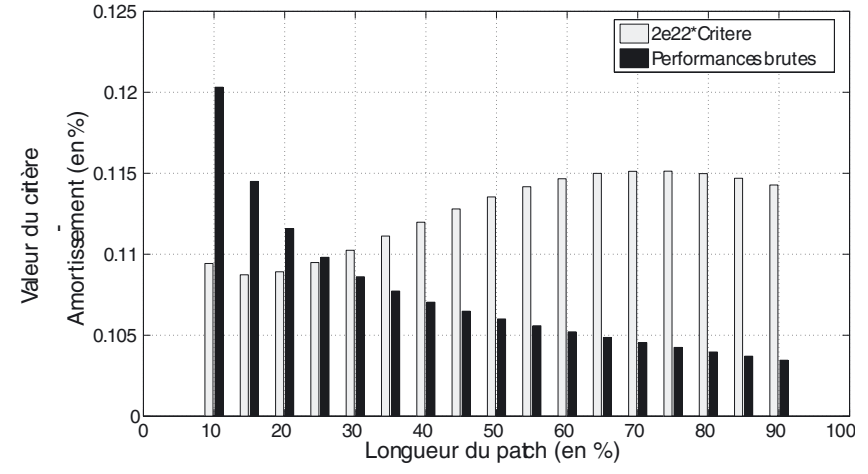

Fig. 10. Comparaison entre les performances brutes et le critère en fonction de la longueur du patch, dans le cas de l'actionnement par cisaillement et des conditions aux limites de type encastrée-libre au mode 1.

Tableau 8. Comparaison entre les performances brutes et les performances optimisées à amortissement constant dans le cas de l'actionnement par cisaillement et des conditions aux limites de type encastrée-libre au mode 1 .

\begin{tabular}{ccc}
\hline $\begin{array}{c}\text { Amortissement } \\
(\text { en } \%)\end{array}$ & $\begin{array}{c}\text { Résistance brute } \\
(\mathrm{k} \Omega)\end{array}$ & $\begin{array}{c}\text { Résistance } \\
\text { optimisée }(\mathrm{k} \Omega)\end{array}$ \\
\hline 0,101 & 8,6 & 5 \\
0,1015 & 12,7 & 8,4 \\
0,102 & 16,9 & 15 \\
\hline
\end{tabular}

\subsubsection{Poutre encastrée-libre}

Dans un premier temps, nous optimisons grâce au critère la longueur du patch piézoélectrique. Sur la figure 10, nous superposons les performances brutes et le critère en fonction de la longueur du patch. La longueur optimale pour les performances brutes est obtenue à $10 \%$ de la longueur totale de la poutre soit $10 \mathrm{~mm}$ et à $75 \%$ de la longueur totale de la poutre soit $75 \mathrm{~mm}$ pour l'optimisation grâce au critère.

Dans un deuxième temps, nous mettons en place la résistance de shunt pour contrôler la structure. Le tableau 8 donne un comparatif de résistances à valeurs constantes d'amortissement du mode 1 pour les deux processus d'optimisation. La structure optimisée montre une diminution de la valeur de la résistance donc de l'énergie de contrôle. Les performances en termes d'amortissement sont faibles pour les cas d'actionnement en cisaillement. Cependant, l'optimisation de ce type de système peut s'avérer utile lorsque une capacité négative est mise en parallèle de la plaquette piézoélectrique. Le couplage électromécanique effectif est alors augmenté de manière significative.

De la même manière, le tableau 9 donne un comparatif de performances à valeur de résistance constante pour les deux optimisations. La stratégie optimisée via le critère (37) donne de meilleurs résultats à résistance égale. 
Tableau 9. Comparaison entre les performances brutes et les performances optimisées à valeur de résistance constante dans le cas de l'actionnement par cisaillement et des conditions aux limites de type encastrée-libre au mode 1.

\begin{tabular}{ccc}
\hline $\begin{array}{c}\text { Résitance } \\
(\mathrm{k} \Omega)\end{array}$ & $\begin{array}{c}\text { Amortissement brut } \\
(\text { en \%) }\end{array}$ & $\begin{array}{c}\text { Amortissement } \\
\text { optimisé (en \%) }\end{array}$ \\
\hline 2 & 0,1002 & 0,1004 \\
5 & 0,10056 & 0,101 \\
7,5 & 0,10087 & 0,1014 \\
10 & 0,10117 & 0,1017 \\
15 & 0,10177 & 0,1019 \\
\hline
\end{tabular}

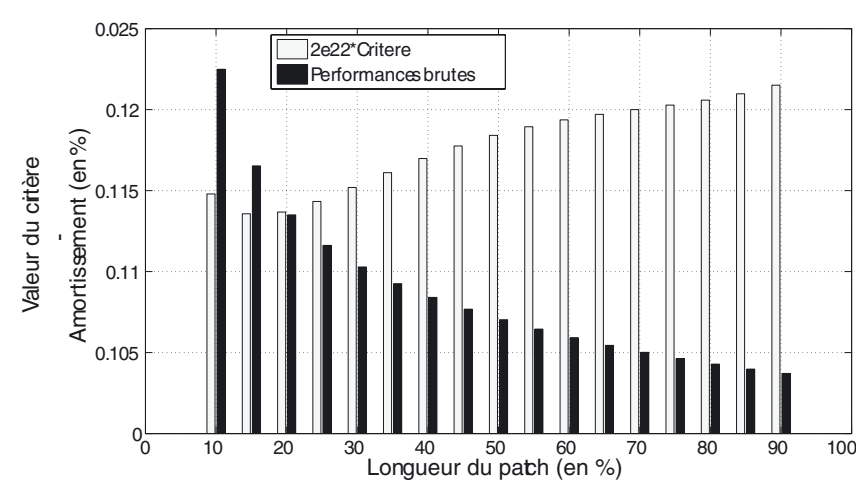

Fig. 11. Comparaison entre les performances brutes et le critère en fonction de la longueur du patch, dans le cas de l'actionnement par cisaillement et des conditions aux limites de type encastrée-guidée au mode 1.

Tableau 10. Comparaison entre les performances brutes et les performances optimisées à amortissement constant dans le cas de l'actionnement par cisaillement et des conditions aux limites de type encastrée-guidée au mode 1 .

\begin{tabular}{ccc}
\hline $\begin{array}{c}\text { Amortissement } \\
(\text { en } \%)\end{array}$ & $\begin{array}{c}\text { Résistance brute } \\
(\mathrm{k} \Omega)\end{array}$ & $\begin{array}{c}\text { Résistance } \\
\text { optimisée }(\mathrm{k} \Omega)\end{array}$ \\
\hline 0,1005 & 2,55 & 1,7 \\
0,101 & 5 & 3,6 \\
0,1015 & 7,25 & 6,45 \\
\hline
\end{tabular}

\subsubsection{Poutre encastrée-guidée}

Tout d'abord, nous optimisons grâce au critère la longueur du patch piézoélectrique. Sur la figure 11, nous superposons les performances brutes et le critère en fonction de la longueur du patch. La longueur optimale pour les performances brutes est obtenue à $10 \%$ de la longueur totale de la poutre soit $10 \mathrm{~mm}$ et à $90 \%$ de la longueur totale de la poutre soit $90 \mathrm{~mm}$ pour l'optimisation grâce au critère.

Ensuite, nous mettons en place la résistance de shunt pour contrôler la structure. Le tableau 10 donne un comparatif de résistances à valeur constante d'amortissement du mode 1 pour les deux optimisations étudiées. La structure optimisée montre une diminution de la valeur de la résistance donc de l'énergie de contrôle.
Tableau 11. Comparaison entre les performances brutes et les performances optimisées à valeur de résistance constante dans le cas de l'actionnement par cisaillement et des conditions aux limites de type encastrée-guidée au mode 1 .

\begin{tabular}{ccc}
\hline $\begin{array}{c}\text { Résitance } \\
(\mathrm{k} \Omega)\end{array}$ & $\begin{array}{c}\text { Amortissement brut } \\
(\text { en \%) }\end{array}$ & $\begin{array}{c}\text { Amortissement } \\
\text { optimisé (en \%) }\end{array}$ \\
\hline 2 & 0,10038 & 0,10059 \\
3,5 & 0,1007 & 0,10098 \\
5 & 0,101 & 0,10129 \\
7,5 & 0,10156 & 0,10162 \\
\hline
\end{tabular}

Enfin, le tableau 11 donne un comparatif de performances à valeur de résistance constante pour les deux optimisations. La structure optimisée via le critère développé amène de performances supérieures, en termes d'amortissement, comparées à celles de l'optimisation via l'amortissement modal.

\section{Conclusions}

Cet article présente le développement et l'application sur un cas simple d'un critère d'optimisation structurale de stratégies de shunt résistif. Ce critère est défini à partir de paramétres de conception simples. Deux séries d'analyse modale sont nécessaires pour les calculer. Les résultats numériques montrent que l'optimisation à l'aide du critère permet de trouver un meilleur compromis entre performances, en termes d'amortissement modal, et valeur de résistance de shunt par rapport à l'optimisation structurale liée à l'amortissement modal simple.

Remerciements. Le premier auteur adresse ses remerciements à la communauté européenne pour son soutien financier à travers le projet CASSEM (contrat n FP6 NMP3-CT-13517).

\section{Références}

[1] M. Lin, F.-K. Chang, The manufacture of composite structures with a built-in network of piezoceramics, Compos. Sci. Tech. 62 (2002) 919-939

[2] W.J. Staszewski, C. Boller, G.R. Tomlinson, Health Monitoring of Aerospace Structures: Smart Sensor Technologies and Signal Processing, Wiley and New York, 2004

[3] Z. Su, X. Wang, W. Chen, L. Ye, D. Wang, A built-in active sensor network for health monitoring of composite structures, Smart Mater. Struct. 15 (2006) 1939-1949

[4] F.E. Scire, C.E. Teague, Piezodriven $50 \mu \mathrm{m}$ range stage with nanometer resolution, Rev. Sci. Instrum. 49 (1978) 1735-1739

[5] S.T. Sith, D.G. Chetwynd, Foundations of ultraprecision mechanism design, Gordon and Breach Science Publishers, 1978 
[6] A.H. Slocum, Precision machine design, Prentice-Hall, Inc., 1992

[7] D.C. Karnopp, A.K. Trikha, Comparative study of optimization techniques for shock and vibration isolation, J. Eng. Ind. Trans. ASME 91 (1969) 1128-1132

[8] C.E. Kaplow, J.R. Velman, Active local vibration isolation applied to a flexible space telescope, J. Guid. Contr. Dynam. 3 (1980) 227-233

[9] R.M. Chalasani, Ride performance potential of active suspension systems, Part 1: Simplified analysis based on a quarter-car model, ASME Symposium on simulation and control of ground vehicles and transportation systems, Anaheim, CA, Dec. 1984

[10] C.R. Fuller, S.J. Elliott, P.A. Nelson, Active Control of Vibration, Academic Press, 1996

[11] Y. Meyer, T. Verdot, M. Collet, J. Baborowski, P. Muralt, Active Isolation of Electronic Micro Components with Piezoelectrically-Transduced Silicon MEMS Devices, Smart Mater. Struct. 16 (2007) 128-134

[12] Y. Meyer, M. Collet, Mixed control for robust vibration isolation: numerical energy comparison for an active micro suspension device, Smart Mater. Struct. 16 (2007) $1361-1369$

[13] L. Meirovitch, Dynamics and control of structures, New-york and Wiley, 1990

[14] A. Preumont, Vibration Control of Active Structures: An Introduction, Kluwer Academic Publishers, Dordrecht/Boston/London, 2002

[15] M.J. Balas, Direct velocity feedback control of large space structures, J. Guid. Contr. Dynam. 2 (1979) 252-253

[16] M. Collet, V. Walter, P. Delobelle, Active Damping of a Micro-Cantilever Piezo-Composite Beam, J. Sound Vib. 260 (2003) 453-476

[17] J.L. Lions, Exact controllability, stabilization and perturbations for distributed systems, SIAM Rev. 30 (1988) $1-68$

[18] E.F. Crawley, J. de Luis, Use piezoelectric actuators as element of intelligent structures, AIAA J. 25 (1987) 13731385

[19] A. Baz, S. Poh, Performance of an active control system with piezoelectric actuators, J. Sound Vib. 126 (1988) 327-342

[20] T. Takagi, M. Sugeno, Fuzzy identification of systems and its applications, IEEE SMC 15 (1985) 116-1322

[21] R.E. Bellman, I.L. Glicksberg, O. Gross, On the bangbang control problem, Rand. Corporation, 1955

[22] N.W. Hagood, E.F. Crawley, Damping of structural vibrations with piezoelectric materials and passive electrical networks, J. Sound Vib. 142 (1991) 243-268

[23] C.L. Davis, G.A. Lesieutre, An actively tuned solid-state vibration absorber using capacitive shunting of piezoelectric stiffness, J. Sound Vib. 232 (2000) 601-617

[24] R.L. Forward, Electromechanical transducer-coupled mechanical structure with negative capacitance compensation circuit, US Patent Specification 4 (1979) 158-787

[25] S. Behrens, A.J. Fleming, S.O.R. Moheimani, A broaband controller for shunt piezoelectric damping of structural vibration, Smart Mater. Struct. 12 (1999) 1-22

[26] S.Y. Wu, Piezoelectric shunts with parallel R-L circuit for smart structural damping and vibration control, In Proc. SPIE Symposium on Smart Structures and Materials Passive Damping and Isolation, 1996, pp. 259-269
[27] J.J. Hollkamp, Mutlimodal passive vibration suppression with piezoelectric materials and resonant shunt, J. Intell. Mater. Syst. Struct. 5 (1994)

[28] S.Y. Wu, Method for multiple mode shunt damping of structural vibration using a single PZT transducer, In Proc. SPIE Symposium on Smart Structures and Materials - Smart Structures and Intelligent Systems (1998) 159-167

[29] W.W. Clark, Vibration control with state-switching piezoelectric materials, J. Intell. Mater. Syst. Struct. 11 (2000) 263-71

[30] L.R. Corr, W.W. Clark, A novel semi-active multi-modal vibration control law for a piezoceramic actuator, VAJ 125 (2003) 214-222

[31] C. Richard, Enhanced semi passive damping using continuous switching of a piezoelectric device on an inductor, In Proc. SPIE Symposium on Smart Structures and Materials - Passive Damping and Isolation, 2000, pp. 288-299

[32] L. Petit, E. Lefeuvre, C. Richard, D. Guyomar, Broadband semi passive piezoelectric technique for structural damping, In Proc. SPIE Symposium on Smart Structures and Materials - Passive Damping and Isolation, 2004, pp. 414-425

[33] E. Lefeuvre, D. Guyomar, L. Petit, C. Richard, A. Badel, Semi passive structural damping by synchronized switching on voltage sources, SMEBA 03, Suzhou, China, May 2004

[34] M. Tawfik, A. Baz, Experimental and spectral finite element study of plates with shunted piezoelectric patches, Int. J. Acoust. Vib. 9 (2004) 87-97

[35] T. Takigami, T. Tomioka, Investigation to suppress bending vibration of railway vehicle carbodies using piezoelectric elements, QR of RTRI 46 (2005) 225-230

[36] M. Neubauer, R. Oleskiewcz, Suppression of brake squeal using shunted piezoceramics, J. Vib. Acoust. 130 (2008) 021005

[37] A. Belloli, P. Ermanni, Optimum placement of piezoelectric ceramic modules for vibration suppression of highly constrained structures, Smart Mater. Struct. 16 (2007) $1662-1671$

[38] M.I. Frecker, Recent advances in optimization of smart structures and actuators, J. Intell. Mater. Syst. Struct. 14 (2003) 207-216

[39] S. Devasia, T. Meressi, B. Paden, E. Bayo, Piezoelectric actuator design for vibration suppression: placement and sizing, J. Guid. Contr. Dynam. 16 (1993) 859-864

[40] H. Kwakernaak, R. Sivan, Linear optimal control systems, Wiley-Interscience, 1972

[41] A.K. Dhingra, B.H. Lee, Optimal placement of actuators in actively controlled structures, Eng. Optim. 23 (1994) 99-118

[42] A. Arbel, Controllability measures and actuator placement in oscillatory systems, Int. J. Contr. 33 (1981) 565574

[43] A. Hac, L. Liu, Sensor and actuator location in motion control of flexible structures, J. Sound Vib. 167 (1993) 239-261

[44] A.K. Dhingra, B.H. Lee, Multiobjective design of actively controlled structures using a hybrid optimization method, Int. Jour. Num. Meth. Eng. 38 (1995) 3380-3401

[45] S. Kondoh, C. Yatomi, K. Inoue, The positioning of sensors and actuators in the vibration control of flexible systems, JSME 33 (1992) 145-152 
[46] S.J. Kim, J.D. Jones, Influence of piezo-actuator thickness on the active vibration control of a cantilever beam, J. Intell. Mater. Syst. Struct. 6 (1995) 610-623

[47] R.A. Meric, S. Saigal, Shape sensitivity analysis of piezoelectric structures by the adjoint variable method, AIAA J. 29 (1991) 1313-1318

[48] J.K. Hwang, C.H. Choi, C.K. Song, J.M. Lee, Robust LQG control of an all-clamped thin plate with piezoelectric actuators/sensors, IEEE/ASME Trans. Mechatron. 2 (1997) 205-212

[49] M. Collet, L. Jezequel, Active control with piezo-electric layers optimization, J. Struct. Control. 1 (1995)

[50] F. Fahroo, Y. Wang, Optimal location of piezoceramic actuators for vibration suppression of a flexible stucture, in Proc. the 36th IEEE Conference on Decision and Control, San Diego, CA, USA, 1997, pp. 1966-1971
[51] S.Y. Yang, W.H. Huang, Is a collocated piezoelectric sensor/actuator pair feasible for an intelligent beam? J. Sound Vib. 216 (1998) 529-538

[52] P. Monnier, M. Collet, J. Piranda, Definition of mechanical design parameters to optimize efficiency of Integral Force Feedback. Struct. Contr. Health Monit. 12 (2005) 65-89

[53] M. Naillon, R.H. Coursant, F. Besnier, Analyse de structures piézoélectriques par une méthode d'éléments finis, Acta Elec. 25 (1983) 341-362

[54] M. Collet, K.A. Cunefare, Modal synthesis and dynamical condensation methods for accurate piezoelectric systems impedance computation, J. Intell. Mater. Syst. Struct., on-line, 2008 